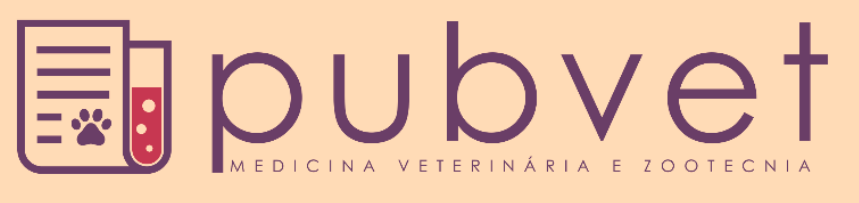

https://doi.org/10.31533/pubvet.v13n2a270.1-9

\title{
Alimentador automático de bezerras: Revisão
}

\author{
Gustavo Rafael de Oliveira Silva ${ }^{1}$, Marcos Aurélio Lopes $^{2}{ }^{\bullet}$, Luiz Marcos Simões Filho ${ }^{3} \bullet$ \\ ${ }^{1}$ Mestre em Produção Animal, Universidade Federal de Lavras, Médico Veterinário Rehagro, Lavras - MG, Brasil. \\ ${ }^{2}$ Professor da Universidade Federal de Lavras, Departamento de Medicina Veterinária. Lavras - MG, Brasil. \\ ${ }^{3}$ Graduado em Medicina Veterinária, Universidade Federal de Minas Gerais, Terra Promissão Agronegócios, Santo Antônio do Amparo-MG, Brasil. \\ *Autor para correspondência, E-mail: malopes@dmv.ufla.br
}

Resumo. Os alimentadores automáticos de bezerras, que foram criados em 1980 na Alemanha, vêm ganhando mais espaço recentemente. São equipamentos que automatizam o processo de fornecimento de leite e concentrado possibilitando maior precisão no controle do consumo individual de alimento líquido e sólido. Objetivou-se, com esta revisão da literatura, reunir informações que esclareçam o funcionamento dessa tecnologia e discutir as suas vantagens e limitações, bem como apresentar resultados de pesquisas sobre o tema, visando auxiliar técnicos e pecuaristas no processo de tomada de decisão sobre a adoção, ou não, da tecnologia. Concluiu-se que os alimentadores automáticos apresentam muitas vantagens tais como: economia com mão de obra, redução do custo alimentar e redução da idade ao desaleitamento com melhor manutenção do peso corporal. No entanto, eles apresentam algumas limitações que precisam ser consideradas como: possibilidade de maior facilidade de transmissão de doenças, em função do sistema de criação ter que ser coletivo, e a necessidade de possuir instalações para que as bezerras fiquem nos primeiros cinco dias de vida. Também é importante ressaltar que, para o sucesso de sua implantação é fundamental uma rotina de análise dos dados gerados pelo equipamento, limpeza criteriosa das instalações e boa observação das bezerras.

Palavras-chave: aleitamento, automação, manejo, zootecnia de precisão

\section{Automatic calf feeder: Review}

\begin{abstract}
The automated calf feeders, created in 1980 in Germany, have been gaining more space recently. It is a device that automates the process of providing milk and starter enabling more precise control of individual consumption of liquid and solid food. It is aimed, with this literature review, to gather information to clarify the operation of this technology and discuss their advantages and limitations, besides to present the researches results about this subject, aiming to help the technicians and farmers in the process of decision about the adoption, or not, of the technology. It was concluded that automatic feeders have many advantages like: economy with labor, reduction of feed costs and reduction of weaning age with better body weight manutention, but it has some limitations that need to be observed like: ease of diseases transmission and the need of a building for calves stay in the five first days of their lives. It is also important to point out, that for the success of this implementation, is fundamental the analysis of collected system data, a careful cleaning of the buildings and a good observation of the calves.
\end{abstract}

Keywords: suckling, automation, management, precision zootechnics

\section{Alimentador automático de terneras: Revisión}

Resumen. Los alimentadores automáticos de terneras, que se crearon en 1980 en Alemania, han ganado más espacio recientemente. Son equipos que automatizan el proceso de 
suministro de leche y concentrado posibilitando mayor precisión en el control del consumo individual de alimento líquido y sólido. Se objetivó, con esta revisión de la literatura, reunir informaciones que esclarezcan el funcionamiento de esa tecnología y discutir sus ventajas y limitaciones, así como presentar resultados de investigaciones sobre el tema, visando auxiliar técnicos y ganaderos en el proceso de toma de decisión sobre la adopción, o no, de la tecnología. Se concluyó que los alimentadores automáticos presentan muchas ventajas tales como: economía con mano de obra, reducción del costo alimentario y reducción de la edad al destete con mejor mantenimiento del peso corporal, pero presentan algunas limitaciones que necesitan ser consideradas como: mayor facilidad de transmisión de enfermedades y la necesidad de poseer instalaciones para que las terneras queden en los primeros cinco días de vida. También es importante resaltar que, para el éxito de su implantación, es fundamental una rutina de análisis de los datos generados por el equipo, limpieza cuidadosa de las instalaciones y buena observación de las terneras.

Palabras clave: lactancia, automatización, manejo, zootecnia de precisión

\section{Introdução}

A automação dos processos dentro da pecuária leiteira se tornou uma tendência, principalmente, em grandes propriedades, sendo importante para viabilizar o aumento da escala de produção. Isso ocorre, uma vez que possibilita elevar a velocidade de execução das tarefas e melhorar a eficiência de utilização de recursos como: mão de obra, alimentos e instalações, podendo, dessa forma, reduzir os custos de produção (Passetti et al., 2016).

O custo com a cria e recria, ou seja, com a criação de fêmeas para reposição do rebanho, representa alto valor dentro da atividade leiteira. Grande parte dele deve-se aos gastos com alimentação e mão de obra que, segundo Santos \& Lopes (2014), ocupam a primeira e segunda colocação, respectivamente, dentro do custo operacional efetivo de produção de fêmeas bovinas leiteiras. Portanto, alternativas para redução dos custos com alimentos e pessoal, em qualquer etapa da criação desses animais, são de grande impacto para a lucratividade do negócio. Na fase de aleitamento das bezerras, a automação do manejo desses animais é uma alternativa para redução de custos e facilitação dos processos.

Nesse contexto, foram desenvolvidos os alimentadores automáticos de bezerras, equipamentos que automatizam o processo de fornecimento de leite, sendo que alguns modelos também automatizam o fornecimento de concentrado para os animais lactentes. Esses equipamentos possibilitam maior precisão no controle do consumo individual de alimento líquido e sólido (Santos, 2013). Objetivou-se, com esta revisão da literatura, reunir informações que esclareçam como funciona a tecnologia e discutir as suas vantagens e limitações, bem como apresentar resultados de pesquisas sobre o tema, visando auxiliar técnicos e pecuaristas no processo de tomada de decisão quanto a adoção, ou não, da tecnologia. Nessa revisão, foram descritos tanto o modelo de alimentador automático que fornece apenas a dieta líquida quanto àqueles mais sofisticadas, que fornecem também o concentrado.

\section{Descrição e funcionamento do equipamento}

Os alimentadores automáticos, criados em 1980 na Alemanha (Heiting, 1992), ainda são pouco utilizados no Brasil, mas em alguns países como na Suécia, por exemplo, a utilização dessa tecnologia já se difundiu bastante e apresentou grande crescimento nos últimos anos (Pettersson et al., 2001). Os equipamentos presentes no mercado variam muito quanto ao preço e grau de sofisticação. Existem alimentadores automáticos mais simples, compostos apenas por uma unidade de alimentação, que permite o fornecimento apenas da dieta líquida, e não oferecem muita flexibilidade para o fornecimento de diferentes quantidades de leite ou sucedâneo para bezerras com diferentes exigências. Esses alimentadores podem ser adquiridos pelo valor de US\$1.600 a US\$2.400. Outros mais sofisticados podem ser adquiridos por US\$ 15.000 a US\$ 18.000. Estes são compostos por duas unidades de alimentação, possuem um software que emite informações sobre o consumo dos animais e permitem o fornecimento de dietas diferentes para cada bezerra (James \& Machado, 2013). Cada unidade de alimentação consegue suportar até 45 bezerras se houver um alto fornecimento de leite por bezerra (12 litros), mas se o fornecimento de leite por bezerra for baixo, é possível alimentar apenas 20 bezerras, porque a quantidade de visitas não recompensadas aumenta e o equipamento perde eficiência (Borderas 
et al., 2009). Contudo, a alocação de 45 bezerras numa única unidade de alimentação é questionável. Segundo Svensson \& Liberg (2006) bezerras mantidas em grupos de 12 a 18 animais tiveram maior incidência de doenças respiratórias (odds ratio $=1,4$ ) do que bezerras criadas em grupos de seis a nove bezerras. Essa constatação permite inferir que quanto maior forem os grupos, maior será o risco de ocorrência de doenças respiratórias, devido ao contato próximo de bezerras de diferentes idades (Maatje et al., 1993).

Basicamente, tanto os alimentadores mais simples quanto os mais sofisticados são compostos por baia de alimentação, um dispositivo para ler o chip de identificação da bezerra, um bico de mamadeira acoplado a um tanque de mistura do leite em pó com água ou de mistura do leite pasteurizado e um software instalado num computador que controla esse equipamento. Os equipamentos mais sofisticados apresentam também um cocho para fornecimento de concentrado (James \& Machado, 2013). Os equipamentos mais sofisticados permitem reduzir automaticamente o fornecimento de leite ou sucedâneo a partir do aumento de consumo de concentrado de forma diferente e individualizada para cada bezerra (James \& Machado, 2013). Esta, inclusive, pode ser considerada uma das grandes vantagens desses alimentadores, uma vez que possibilitam maior controle, por parte do cuidador das bezerras, do consumo, tanto da dieta líquida quanto da sólida. Além disso, alguns desses equipamentos têm funções extras que permitem adicionar medicamentos líquidos ou sólidos à dieta que vai ser fornecida para bezerras doentes, e também possuem a função de limpeza automática dos bicos e tanques de mistura do leite (James \& Machado, 2013).

Nos casos em que se utiliza sucedâneo, ele é automaticamente diluído e misturado à água no tanque de mistura nas proporções determinadas. Adicionalmente, a temperatura do sucedâneo ou do leite fornecido às bezerras também pode ser escolhida e controlada pelo operador do equipamento. Contudo, é importante o monitoramento semanal da temperatura e teor de sólidos do leite para checar o funcionamento do equipamento (James \& Machado, 2013). Segundo Tayler \& Lonsdale (1969), o fornecimento da dieta líquida nas temperaturas de $8{ }^{\circ} \mathrm{C}$ ou $38{ }^{\circ} \mathrm{C}$, desde que constante em todo o período de aleitamento, não interferem no ganho de peso e consumo dos animais. No entanto, Abe et al. (1979) sugerem que a temperatura ideal para o fornecimento de substititutos de leite às bezerras deve estar entre $38,9^{\circ}$ e $41,7^{\circ} \mathrm{C}$. Segundo os autores, é preciso ter em mente que a temperatura corporal normal de uma bezerra saudável é em torno de $38,9^{\circ} \mathrm{C}$. Portanto, o fornecimento de leite frio fará com que a bezerra necessite aquecê-lo em seu organismo após o consumo. Esta situação não apenas promove desperdício de energia, como também pode ser observada notória redução na motivação do animal em consumir o leite.

As bezerras são identificadas com um chip na orelha e toda vez que entram na baia de alimentação, voluntariamente, um dispositivo faz a leitura e envia as informações daquele animal para o software que controla o alimentador. Então, será liberada a quantidade de concentrado e de dieta líquida definida para aquela bezerra e serão coletadas informações sobre a dieta consumida, que ficarão registradas (James \& Machado, 2013). O esquema de um alimentador automático de bezerras pode ser visualizado na figura 1.

A bezerra é permitida uma quantidade limitada de visitas ao alimentador e intervalo mínimo entre refeições, que dependerão de alguns pontos. A bezerra é cadastrada no sistema com permissão para ingerir determinada quantidade de leite por dia. O software faz o cálculo de quanto de leite ela poderá ingerir por hora e, levando em consideração a quantidade mínima de leite oferecida pelo equipamento por visita (geralmente, 1,3 litros de leite ou sucedâneo), tem-se o intervalo mínimo de visitas ao alimentador. Exemplo: uma bezerra que ingere nove litros por dia pode ingerir nove litros $/ 24$ horas = 0,375 litros/hora. Assim, o intervalo mínimo de alimentação dela será 1,3 litros/0,375 litros=3,46 horas = três horas e 28 minutos, ou seja, essa bezerra pode se alimentar a cada três horas e 28 minutos. Contudo, normalmente, toda bezerra faz de quatro a cinco visitas por dia ao alimentador, já que, na verdade, elas consomem mais do que 1,3 litros por visita. A quantidade de sólidos proveniente do leite recomendada por bezerra/dia é de 680 a 1.225g (James \& Machado, 2013). De acordo com Drackley (2008), $1 \mathrm{~kg}$ de sólidos do leite possui cerca de 5,37 Mcal de energia, e a exigência de uma bezerra de $45 \mathrm{~kg}$ de peso vivo, é em torno de $1,75 \mathrm{Mcal} / \mathrm{dia}$ na zona termo neutra $\left(15 \mathrm{a} 26^{\circ} \mathrm{C}\right)$, para mantença em condições normais. Segundo Drackley (2008), uma bezerra, para obter energia metabolizável suficiente para ganhar peso, necessita consumir acima de $326 \mathrm{~g}$ de sólidos do leite por dia $(1,75 / 5,37=326 \mathrm{~g})$. Ou seja, se o leite possui $12,5 \%$ de sólidos, a bezerra de $45 \mathrm{~kg}$ de peso vivo, para ganhar peso, em zona 
termo neutra e em condições de normalidade corporal e sanitária, necessita consumir acima de 2,6 litros de leite por dia $(2,6$ litros x $12,5 \%=326 \mathrm{~g})$. Se a bezerra, portanto, não necessitar debelar qualquer infecção ou doença, ou ainda, se não precisar enfrentar qualquer situação de estresse, qualquer volume de leite acima dos 2,6 litros, a permitirá crescer e ganhar peso. No entanto, a exigência de mantença pode ser aumentada significativamente em casos de doenças e/ou em casos de estresse térmico, podendo muitas vezes ser até 50\% maior em casos mais severos (Shearer \& Beede, 1990). Mas Drackley (2008) faz ainda uma recomendação, para que não faltem nutrientes à bezerra para manter e crescer: na primeira semana de vida, $1,5 \%$ do peso vivo ao dia em sólidos do leite; e da segunda semana de vida em diante, $2 \%$ do peso vivo ao dia em sólidos do leite.

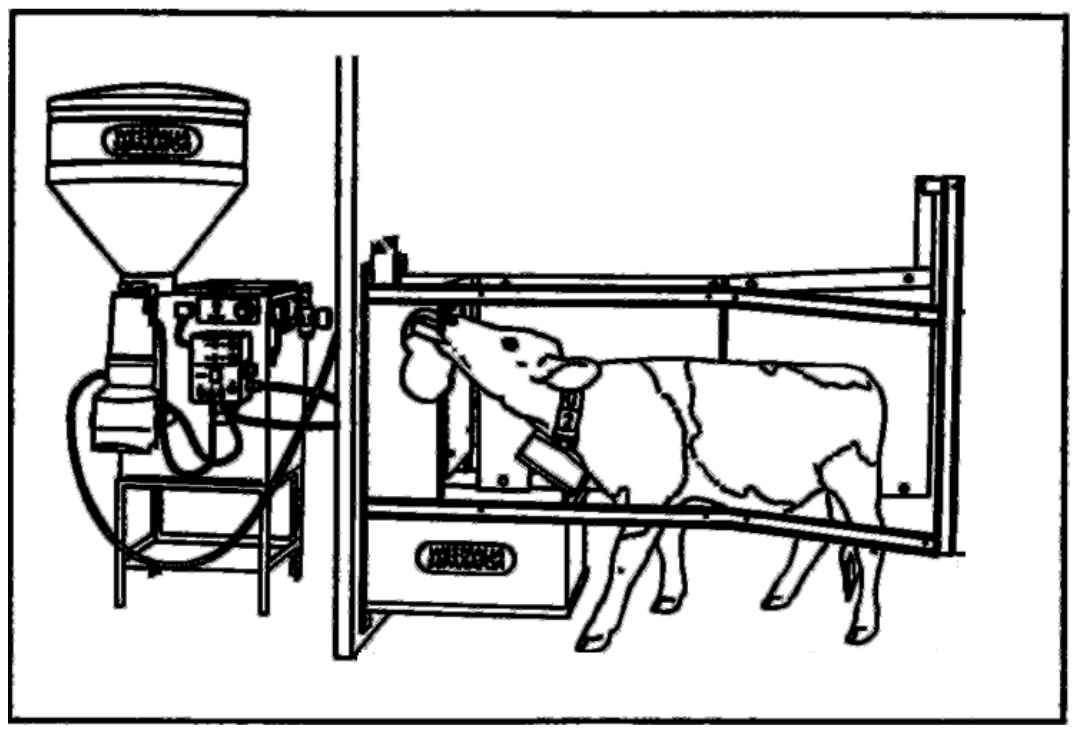

Figura 1. Visão do alimentador automático (Kung Junior et al., 1997).

Nos primeiros cinco dias de vida, as bezerras ficam no bezerreiro individual para que se possa assegurar que elas estão se alimentando bem. Então, após esse período, elas são transferidas para galpões onde terão acesso aos alimentadores automáticos, sendo divididas em lotes por idade, da forma mais homogênea possível. Esses lotes devem ser formados respeitando-se a quantidade máxima de 20 a 45 bezerras por estação de alimentação. O ideal é que as bezerras não tenham diferenças de idade maior do que três semanas, pois isso pode fazer com que as mais jovens não consigam competir com as maiores e ingiram menos leite do que necessitam. No dia da transferência das bezerras para esses lotes, elas não recebem leite ou sucedâneo pela manhã para que tenham mais vontade de procurar o alimentador e ingerir a dieta líquida (James \& Machado, 2013).

Os alimentadores automáticos mais sofisticados são dotados de dispositivo que os permitem oferecer às bezerras também, além do leite, alimentos concentrados. O consumo de concentrado pode variar muito entre as bezerras, muito embora, segundo Drackley et al. (2006) e Drackley (2008), apesar de as bezerras conseguirem já consumir alimentos sólidos desde os 14 dias de vida, o consumo de concentrado somente será em quantidades energéticas suficientes após os 30 dias de vida. Khan et al. (2007), no entanto, apontam que o consumo de concentrado ainda no início da vida das bezerras é fator fundamental para um melhor desenvolvimento do rúmen e de suas papilas. Ainda, segundo Khan et al. (2007), bezerras que receberam $25 \%$ do peso corporal em litros de leite/dia por 30 dias, e na sequência, $10 \%$ do peso corporal em litros de leite/dia até os 60 dias de vida, tiveram maior consumo de alimento concentrado e, consequentemente, melhor desenvolvimento dos pré-estômagos do que bezerras que receberam o volume de $10 \%$ do peso corporal durante todo o período de aleitamento (Tabela 1). O desenvolvimento adequado do rúmen, ainda no período de aleitamento, é fator crucial para uma boa passagem pelo processo de desaleitamento e redução máxima de riscos de perda de desempenho das bezerras no período subsequente, quando já estarão totalmente desaleitadas e há comumente significativa redução em ganho de peso ou muitas vezes até perda de peso. Dentro deste ponto estratégico e fundamental para as fazendas, talvez seja onde resida uma das mais importantes vantagens do uso dos alimentadores automáticos. 
Tabela 1. Desenvolvimento ruminal e consumo de concentrado de bezerras em diferentes dietas líquidas

\begin{tabular}{lcc}
\hline Parâmetros & $\begin{array}{c}\text { Dieta convencional 10\% do } \\
\text { PC em leite }\end{array}$ & $\begin{array}{c}\text { Dieta 25\% do PC em leite até 30 dias e } 10 \% \text { do } \\
\text { PC em leite até } 60 \text { dias }\end{array}$ \\
\hline Peso do rúmen $(\mathrm{kg})$ & $1,37 \mathrm{~b}$ & $1,89 \mathrm{a}$ \\
Peso do retículo $(\mathrm{kg})$ & $0,18 \mathrm{~b}$ & $0,29 \mathrm{a}$ \\
Peso do omaso $(\mathrm{kg})$ & $0,53 \mathrm{~b}$ & $0,68 \mathrm{a}$ \\
Peso do abomaso $(\mathrm{kg})$ & $0,57 \mathrm{~b}$ & $0,71 \mathrm{a}$ \\
Espessura parede do rúmen $(\mathrm{cm})$ & $1,15 \mathrm{~b}$ & $1,47 \mathrm{a}$ \\
Altura das papilas $(\mathrm{cm})$ & $0,71 \mathrm{~b}$ & $0,96 \mathrm{a}$ \\
Concentração de papilas/cm & $71,00 \mathrm{~b}$ & $86,00 \mathrm{a}$ \\
Consumo de concentrado pré-desmama & $400,00 \mathrm{~b}$ & $511,61 \mathrm{a}$ \\
Consumo de concentrado pós-desmama & $1534,38 \mathrm{~b}$ & $2086,88 \mathrm{a}$ \\
\hline
\end{tabular}

Fonte: Adaptado de Khan et al. (2007).

\section{Vantagens da utilização}

O uso dessa tecnologia traz várias vantagens ao produtor como economia com mão de obra, redução no custo de alimentação, redução da idade ao desaleitamento com melhor manutenção do peso corporal, auxílio na identificação de doenças, menor taxa de mortalidade, redução da mamada cruzada, possibilidade de manter bezerras com diferentes necessidades no mesmo lote e permite que haja maior interação social entre as bezerras.

Kung Junior et al. (1997) relataram que a média de tempo gasto pelos funcionários para manejar bezerras num sistema convencional é de 10 minutos/bezerra/dia, enquanto que para bezerras criadas através de alimentadores automáticos, se gasta menos de um minuto/bezerra/dia. Assim, esses pesquisadores estimaram que se paga o investimento no alimentador automático em dois ou três anos considerando a economia com mão de obra. Para esse cálculo foi considerado um rebanho de 200 vacas em lactação, com taxa de descarte de $35 \%$ e taxa de mortalidade de bezerras de $10 \%$, necessitando, portanto, de 77 bezerras/ano. O equipamento utilizado no estudo foi o da Westfalia Systemat, cujo valor de aquisição foi de US\$12.000,00. Tal equipamento era composto por duas estações de alimentação que suportavam 25 bezerras cada uma (50 bezerras no total) e fornecia leite ou sucedâneo e concentrado. Portanto, 77 bezerras x oito semanas (tempo de aleitamento) x sete dias/semana x 10 minutos/dia (economia de mão de obra/bezerra/dia) $=719$ horas/ano x US\$7/hora (preço da mão de obra considerado) $=$ US\$ 5.033,00 de economia com mão de obra por ano. Logo, US\$ $12.000,00 \div$ US\$ $5.033,00=2,3$ anos para se pagar o investimento. James \& Machado (2013) também citam a redução de mão de obra como uma vantagem dos alimentadores automáticos, mas alertam para a necessidade de gastar-se tempo monitorando o funcionamento do equipamento e sua higienização, além de avaliar consumo e saúde dos animais rotineiramente, andando-se entre as bezerras para observá-las e diagnosticar precocemente as doenças. A importância dessas ações pode ser comprovada pela conclusão de Winder et al. (2016), que relacionaram melhor qualidade de mão de obra com redução de risco de mortalidade de bezerras alimentadas por alimentadores automáticos.

Sabe-se que a bezerra apresenta um comportamento ingestivo diferente (Passillé \& Rushen, 2012). Com base nesse fato, de acordo com a variação individual dos animais, à medida que a bezerra aumenta o consumo da dieta sólida, pode-se reduzir o fornecimento da dieta líquida diminuindo os custos da criação da bezerra, já que alimentar as bezerras com leite e sucedâneo é mais caro do que alimentá-las com concentrado (Passillé \& Rushen, 2016). Uma das vantagens tidas como principais, portanto, do uso do alimentador automático, é justamente neste tema, pois o próprio alimentador automático pode ser parametrizado para reduzir o fornecimento de leite gradativamente à medida que o consumo de concentrado sobe (o consumo de concentrado é medido diariamente pelo próprio sistema operacional do alimentador automático). Segundo Roth et al. (2009), bezerras desaleitadas por meio do método concentrado - dependente custam US\$128,30, enquanto que aquelas desaleitadas utilizando métodos convencionais custam US\$149,00, representando economia de US $\$ 20,70$ por bezerra.

Alguns sistemas de criação de bezerras têm funcionado da seguinte forma: todas as bezerras começam sua vida recebendo a mesma quantidade de sucedâneo ou leite: 12 litros/dia. A partir do momento que elas apresentam um consumo de concentrado de $200 \mathrm{~g} /$ dia por três dias seguidos, inicia a 
redução do fornecimento de dieta líquida para essa bezerra, e quando atingem o consumo de $1.400 \mathrm{~g} / \mathrm{dia}$ de concentrado por três dias seguidos é feito o desaleitamento. Dessa forma, tem-se conseguido redução de 13 a 14 dias no período de aleitamento das bezerras, sem diminuir o ganho de peso desses animais (Passillé \& Rushen, 2016).

A possibilidade de ajustar a idade do desmame para a bezerra, de acordo com o consumo de concentrado, faz com que as bezerras consigam manter o ganho de peso na fase do pós desmame, especialmente porque, dessa forma, segundo Khan et al. (2007), com uma adequada evolução no consumo de concentrado e iniciando-se a sua oferta o mais cedo possível (cerca de 14 dias de vida), o que se tem é um ótimo desenvolvimento dos pré-estômagos, bem como das papilas ruminais. Segundo Passillé \& Rushen (2016), bezerras desaleitadas utilizando-se alimentadores automáticos aos 75,8 $( \pm 10,7)$ dias apresentam maior peso corporal na $10^{\mathrm{a}}$ e $13^{\mathrm{a}}$ semana de vida do que bezerras desaleitadas precocemente numa idade fixa de 48 dias pelos métodos convencionais, e mesmo peso na $13^{\mathrm{a}}$ semana do que bezerras desaleitadas numa idade fixa de 89 dias, também pelos métodos convencionais, só que com um custo mais baixo, já que há menor gasto de leite e sucedâneo (Tabela 2). Entretanto, Maatje et al. (1993) observaram menor consumo de sucedâneo e menor taxa de crescimento em bezerras criadas em grupos de 15 animais com alimentador automático, comparativamente a bezerras criadas em sistema convencional. De acordo com Roth et al. (2009), a medida do consumo de concentrado pelas bezerras, feito pelos alimentadores automáticos, é uma boa ferramenta para detecção precoce de doenças. Eles atribuíram notas ao estado de saúde de cada animal e observaram correlação entre o baixo consumo de concentrado e a ocorrência de doenças nas bezerras. A quantidade de visitas não recompensadas ao alimentador também é um indicador da saúde das bezerras, uma vez que bezerras sadias fizeram mais visitas não recompensadas ao alimentador do que animais doentes (Svensson \& Jensen, 2007). Portanto, se bem usados, os dados do software podem auxiliar no diagnóstico de doenças das bezerras.

Tabela 2. Peso (média \pm desvio padrão, $\mathrm{kg}$ ) em cada semana de idade de bezerras desaleitadas pelos métodos precoce (48 dias), tardio (89 dias) ou de acordo com o consumo de concentrado $(75,8 \pm 10,7)$ dias)

\begin{tabular}{lccc}
\hline Semana & Precoce & Tardio & Consumo de concentrado \\
\hline 3 & $53,6 \pm 1,8$ & $50,6 \pm 2,0$ & $52,4 \pm 1,3$ \\
6 & $68,1 \pm 1,4$ & $69,3 \pm 1,6$ & $68,3 \pm 1,0$ \\
8 & $73,4 \pm 2,3^{\mathrm{A}}$ & $82,1 \pm 2,6^{\mathrm{B}}$ & $77,3 \pm 1,6^{\mathrm{AB}}$ \\
10 & $84,5 \pm 2,4^{\mathrm{A}}$ & $94,9 \pm 2,6^{\mathrm{B}}$ & $90,6 \pm 1,6^{\mathrm{B}}$ \\
12 & $100,2 \pm 2,9^{\mathrm{A}}$ & $106,6 \pm 3,3^{\mathrm{A}}$ & $108,0 \pm 2,1^{\mathrm{B}}$ \\
13 & $108,2 \pm 2,8^{\mathrm{A}}$ & $115,9 \pm 3,1^{\mathrm{AB}}$ & $115,2 \pm 2,0^{\mathrm{B}}$ \\
\hline
\end{tabular}

* Letras diferentes na mesma linha indicam diferença significativa $(\mathrm{P}<0,05)$. Fonte: Passillé \& Rushen (2016).

Além disso, o aumento da frequência de fornecimento de leite, permitido pelos alimentadores automáticos, sem aumentar necessidade de mão de obra, resulta em menor taxa de mortalidade de animais até o primeiro parto (Kmicikewycz et al., 2013). A mamada cruzada também não é um problema dos alimentadores automáticos, uma vez que pode utilizar-se da estratégia de reduzir o fluxo de leite no bico para que as bezerras mamem mais tempo e satisfaçam sua vontade de sugar algo (Jensen, 2003).

Esses equipamentos, por possuírem boa precisão na medida e controle do consumo de leite ou sucedâneo e concentrado, possibilitam que bezerras com diferentes exigências fiquem no mesmo lote, facilitando o manejo. Entretanto, é preciso esclarecer que não pode haver grandes diferenças de idade, pois, nesse caso, as bezerras menores poderiam ser prejudicadas (Roth et al., 2008). Segundo Sowerby \& Polan (1978), experiências prévias de agrupamento reduzem problemas associados à integração de animais adultos. Portanto, como as bezerras criadas em alimentadores automáticos estão sujeitas a constantes interações com diferentes bezerras, isso faz com que elas se adaptem melhor às trocas de lotes na vida adulta. Vacas com experiências sociais prévias estabelecem uma hierarquia em 0 a 2 dias após o agrupamento. Animais sem essas experiências gastam de 2 a 4 dias Kondo \& Hurnik (1990). De acordo com Brakel \& Leis (1976), os efeitos negativos na produção de leite podem durar de 1 a 2 semanas, além dos acidentes e mortes de animais que podem ocorrer, causando prejuízos ao produtor. 


\section{Limitações da utilização}

A criação de bezerras em alimentadores automáticos também possui algumas desvantagens. Entre elas, a necessidade de que as bezerras sejam criadas de forma individual nos primeiros dias, o que representa certo complicador de manejo e gasto com instalações (James \& Machado, 2013). Muito embora o volume de sistemas de criação de bezerras com a utilização de alimentadores automáticos esteja aumentando ao redor do mundo, Medrano-Galarza et al. (2017) observam que alguns produtores estão desistindo e aposentando seus equipamentos. Os autores relatam que muitos produtores têm encontrado grande dificuldade em manter níveis adequados de sanidade nos bezerreiros, em função da necessidade de se manter as bezerras em sistemas coletivos para a adequada otimização do uso dos alimentadores automáticos. Outros autores, inclusive, já relatam fortes recomendações veterinárias no sentido de se retornar dos sistemas de criação de bezerras coletivos para os individuais (Callan \& Garry, 2002; Paula et al., 2008). O objetivo, portanto, é, de fato, limitar totalmente o contato físico entre os animais, o que poderia facilitar, segundo Callan \& Garry (2002) e Paula et al. (2008), a transmissão de doenças, em especial doenças respiratórias e diarreias.

Com a utilização desses equipamentos, as bezerras são criadas em grupos, o que pode facilitar a transmissão de doenças (Warnick et al., 1977). Por isso, é importante uma boa higiene dos equipamentos e boa observação dos animais para diagnóstico precoce de doenças (James \& Machado, 2013). Segundo Svensson et al. (2003), bezerras criadas em grupos de seis a 30 animais, alimentadas com o alimentador automático, tiveram maior risco (odds ratio $=2,2$ ) de apresentarem doença respiratória do que bezerras criadas individualmente e não houve diferenças em relação à incidência de diarreia (Svensson et al., 2000). Na Suécia, entre os anos de 1980 e 1998, houve aumento de 0,8\% para 7,0\% na incidência de doenças respiratórias, que, segundo (Svensson et al., 2000) pode ser explicado pela maior utilização de sistemas de criação de bezerras em grupos. Medrano-Galarza et al. (2018), em contraposição a outros estudos, encontraram resultados de prevalência de doenças respiratórias e diarreias em bezerras criadas em sistemas coletivos e com a utilização de alimentadores automáticos, muito similares àqueles encontrados em relatos de estudos realizados em fazendas com sistemas de criação de bezerras individualizadas. De acordo com Medrano-Galarza et al. (2018), a utilização de alimentadores automáticos em bezerreiros coletivos, não necessariamente tornam este sistema de criação mais perigoso ou pior do que os sistemas individualizados, sob o ponto de vista da sanidade, sendo a saúde das bezerras muito mais dependente do manejo conferido ao sistema do que do sistema de criação propriamente dito. Eles salientam que a limpeza do equipamento é fundamental, devendo, portanto, ser programado para limpeza automática no mínimo 3 vezes ao dia. Este aspecto foi associado com redução na prevalência de diarreias em bezerreiros testados. Ainda segundo os mesmos autores, o fornecimento de leite integral natural, ao invés de sucedâneos, também foi associado a uma redução na prevalência de doenças respiratórias. E, por fim, duas outras estratégias que os autores comentam, e que foram associadas à reduções na prevalência de doenças, são: introdução dos animais ao sistema coletivo antes dos 8 dias de vida e fornecimento de vitamina E, selênio e probióticos às bezerras.

Além disso, ainda no âmbito das possíveis desvantagens, existe grande diferença em relação ao tempo que as bezerras gastam para fazer a primeira visita sem auxílio ao alimentador depois que são introduzidas nos grupos. Segundo Fujiwara et al. (2014), menos de um terço das bezerras consegue ingerir leite sozinhas através do alimentador automático nas primeiras 24 horas e, aproximadamente, metade delas gastam mais de três dias. Já em experimento realizado por Morita et al. (1999), sete de 26 bezerras precisaram de assistência humana para utilizar o alimentador automático no terceiro dia de utilização do equipamento.

\section{Considerações finais}

Os alimentadores automáticos apresentam muitas vantagens tais como: economia com mão de obra, redução do custo de alimentação, redução da idade ao desaleitamento com melhor manutenção do peso corporal, auxílio na identificação de doenças, menor taxa de mortalidade, redução da mamada cruzada, possibilidade de manter bezerras com diferentes necessidades no mesmo lote e permite que haja maior interação social entre as bezerras. Contudo, apresentam também algumas limitações como: uma maior facilidade de transmissão de doenças, a necessidade de possuir instalações para que as bezerras fiquem nos primeiros cinco dias de vida e o fato de algumas bezerras gastarem um tempo longo para fazer a 
primeira visita ao alimentador sem auxílio. Além disso, é importante ressaltar que, para o sucesso de sua implantação, numa fazenda, é fundamental uma rotina de análise dos dados gerados pelo equipamento, limpeza criteriosa das instalações e boa observação das bezerras pelos tratadores. Apesar de já haver alguns estudos sobre economia com mão de obra e alimentação a partir do uso dos alimentadores automáticos, ainda faltam trabalhos que abordem o retorno econômico dessa tecnologia considerando todas as suas vantagens e desvantagens.

\section{Referências bibliográficas}

Abe, M., Iriki, T., Kondoh, K. \& Shibui, H. (1979). Effects of nipple or bucket feeding of milk-substitute on rumen by-pass and on rate of passage in calves. British Journal of Nutrition, 41(1):175-181.

Borderas, T. F., Passillé, A. M. B. \& Rushen, J. (2009). Feeding behavior of calves fed small or large amounts of milk. Journal of Dairy Science, 92(6):2843-2852.

Brakel, W. J. \& Leis, R. A. (1976). Impact of social disorganization on behavior, milk yield, and body weight of dairy cows. Journal of Dairy Science, 59(4):716-721.

Callan, R. J. \& Garry, F. B. (2002). Biosecurity and bovine respiratory disease. Veterinary Clinics of North America: Food Animal Practice, 18(1):57-77.

Drackley, J. K. (2008). Calf nutrition from birth to breeding. Veterinary Clinics of North America: Food Animal Practice, 24(1):55-86.

Drackley, J. K., Donkin, S. S. \& Reynolds, C. K. (2006). Major advances in fundamental dairy cattle nutrition. Journal of Dairy Science, 89(4):1324-1336.

Fujiwara, M., Rushen, J. \& Passillé, A. M. (2014). Dairy calves' adaptation to group housing with automated feeders. Applied Animal Behaviour Science, 158:1-7.

Heiting, N. (1992). Verfügbare Tränkemittel und ihre Verabreichung über Tränkeautomaten. KTBLSchrift, 35292-100.

James, B. \& Machado, K. (2013). Group housing and feeding systems for calves - opportunities and challenges. Department of Dairy Science Virginia Technology, 1:1-9.

Jensen, M. B. (2003). The effects of feeding method, milk allowance and social factors on milk feeding behaviour and cross-sucking in group housed dairy calves. Applied Animal Behaviour Science, 80(3):191-206.

Khan, M. A., Lee, H. J., Lee, W. S., Kim, H. S., Ki, K. S., Hur, T. Y., Choi, Y. J. (2007). Structural growth, rumen development, and metabolic and immune responses of Holstein male calves fed milk through step-down and conventional methods. Journal of Dairy Science, 90(7):3376-3387.

Kmicikewycz, A. D., da Silva, D. N. L., Linn, J. G. \& Litherland, N. B. (2013). Effects of milk replacer program fed 2 or 4 times daily on nutrient intake and calf growth. Journal of Dairy Science, 96(2):1125-1134.

Kondo, S. \& Hurnik, J. F. (1990). Stabilization of social hierarchy in dairy cows. Applied Animal Behaviour Science, 27(4):287-297.

Kung Junior, L., Demarco, S., Siebenson, L. N., Joyner, E., Haenlein, G. F. W. \& Morris, R. M. (1997). An evaluation of two management systems for rearing calves fed milk replacer. Journal of Dairy Science, 80(10):2529-2533.

Maatje, K., Verhoeff, J., Kremer, W. D. \& Cruijsen, A. L. (1993). Automated feeding of milk replacer and health control of group-housed veal calves. The Veterinary Record, 133(11):266-270.

Medrano-Galarza, C., LeBlanc, S. J., Jones-Bitton, A., DeVries, T. J., Rushen, J., Passillé, A. M., . . . Haley, D. B. (2018). Associations between management practices and within-pen prevalence of calf diarrhea and respiratory disease on dairy farms using automated milk feeders. Journal of Dairy Science, 101(3):2293-2308.

Medrano-Galarza, C., LeBlanc, S. J., Jones-Bitton, A., DeVries, T. J., Rushen, J., Passillé, A. M. \& Haley, D. B. (2017). Producer perceptions of manual and automated milk feeding systems for dairy calves in Canada. Canadian Journal of Animal Science, 98(2):250-259. 
Morita, S., Sugita, S., Yamamoto, M., Hoshiba, S. \& Uemura, T. (1999). Behavioral investigation of group rearing calves in automatic milk replacer feeding system. Nihon Chikusan Gakkaiho, 70(6):542-546.

Passetti, R. A. C., Eiras, C. E., Gomes, L. C., Santos, J. F. \& Prado, I. N. (2016). Intensive dairy farming systems from Holland and Brazil: SWOT analyse comparison. Acta Scientiarum. Animal Sciences, 38(4):439-446.

Passillé, A. M. \& Rushen, J. (2012). Adjusting the weaning age of calves fed by automated feeders according to individual intakes of solid feed. Journal of Dairy Science, 95(9):5292-5298.

Passillé, A. M. \& Rushen, J. (2016). Using automated feeders to wean calves fed large amounts of milk according to their ability to eat solid feed. Journal of Dairy Science, 99(5):3578-3583.

Paula, V. A., Guesdon, V., Passillé, A. M., Von Keyserlingk, M. A. G. \& Weary, D. M. (2008). Behavioural indicators of hunger in dairy calves. Applied Animal Behaviour Science, 109(2-4):180189.

Pettersson, K., Svensson, C. \& Liberg, P. (2001). Housing, feeding and management of calves and replacement heifers in Swedish dairy herds. Acta Veterinaria Scandinavica, 42(4):465-478.

Roth, B. A., Hillmann, E., Stauffacher, M. \& Keil, N. M. (2008). Improved weaning reduces crosssucking and may improve weight gain in dairy calves. Applied Animal Behaviour Science, 111(34):251-261.

Roth, B. A., Keil, N. M., Gygax, L. \& Hillmann, E. (2009). Influence of weaning method on health status and rumen development in dairy calves. Journal of Dairy Science, 92(2):645-656.

Santos, G. \& Lopes, M. A. (2014). Indicadores econômicos de sistemas de produção de leite em confinamento total com alto volume de produção diária. Ciência Animal Brasileira, 15(3):239-248.

Santos, J. A. (2013). Aleitador automático facilita criação. Revista Balde Branco, 40-42.

Shearer, J. K. \& Beede, D. K. (1990). Thermoregulation and physiological responses of dairy cattle in hot weather. Agri-Practice, 11(4):5-17.

Sowerby, M. E. \& Polan, C. E. (1978). Milk production response to shifting cows between intraherd groups. Journal of Dairy Science, 61(4):455-460.

Svensson, C., Emanuelsson, U. \& Pettersson, K. (2000). Health status of dairy calves kept in individual pens or in group pens with or without automatic milk feeder. Paper presented at the Proceedings of the 10th International congress on animal hygiene, Maastricht.

Svensson, C. \& Jensen, M. B. (2007). Identification of diseased calves by use of data from automatic milk feeders. Journal of Dairy Science, 90(2):994-997.

Svensson, C. \& Liberg, P. (2006). The effect of group size on health and growth rate of Swedish dairy calves housed in pens with automatic milk-feeders. Preventive Veterinary Medicine, 73(1):43-53.

Svensson, C., Lundborg, K., Emanuelson, U. \& Olsson, S.-O. (2003). Morbidity in Swedish dairy calves from birth to 90 days of age and individual calf-level risk factors for infectious diseases. Preventive Veterinary Medicine, 58(3-4):179-197.

Tayler, J. C. \& Lonsdale, C. R. (1969). The artificial rearing of calves and their growth on grass diets: I. The effect of type and temperature of milk substitute given ad libitum. The Journal of Agricultural Science, 73(2):279-287.

Warnick, V. D., Arave, C. W. \& Mickelsen, C. H. (1977). Effects of group, individual, and isolated rearing of calves on weight gain and behavior. Journal of Dairy Science, 60(6):947-953.

Winder, C. B., Kelton, D. F. \& Duffield, T. F. (2016). Mortality risk factors for calves entering a multilocation white veal farm in Ontario, Canada. Journal of Dairy Science, 99(12):10174-10181.

Recebido: 14 de janeiro, 2019.

Aprovado: 6 fevereiro, 2019.

Publicado: 26 fevereiro, 2019

Licenciamento: Este artigo é publicado na modalidade Acesso Aberto sob a licença Creative Commons Atribuição 4.0 (CC-BY 4.0), a qual permite uso irrestrito, distribuição, reprodução em qualquer meio, desde que o autor e a fonte sejam devidamente creditados. 